\title{
Carbon star survey of Local Group galaxies
}

\section{The spheroidal galaxy NGC $185^{\star}$}

\author{
P. Battinelli ${ }^{1}$ and S. Demers ${ }^{2}$ \\ 1 INAF, Osservatorio Astronomico di Roma, Viale del Parco Mellini 84, 00136 Roma, Italia \\ 2 Département de Physique, Université de Montréal, CP 6128, Succursale Centre-Ville, Montréal, Québec, H3C 3J7, Canada \\ e-mail: demers@astro.umontreal.ca
}

Received 2 September 2003 / Accepted 15 December 2003

\begin{abstract}
We used the CFH12K wide field camera to survey the carbon star population of NGC 185 using the CN-TiO technique. $145 \mathrm{C}$ stars are identified with a mean $\langle I\rangle=19.99 \pm 0.05$, corresponding to $\left\langle M_{I}\right\rangle=-4.41 \pm 0.05$, a mean luminosity similar to what was recently found for the C star population of NGC 147. The stellar surface density profile of NGC 185 follows a power law with an exponential scale length of $2.53 \pm 0.07^{\prime}$. The surface density of C stars follows also a power law but with a smaller scale length, $1.56 \pm 0.06$, demonstrating that the intermediate-age population of NGC 185 is more concentrated than its old population. A tidal radius, $r_{\mathrm{t}}=22.5 \pm 2.2^{\prime}$ is determined from red giant star counts. The C/M ratio of NGC 185 is $0.17 \pm 0.02$ with no apparent sign of a radial gradient. We compare the carbon star populations of NGC 185 and NGC 147 to conclude that it is unlikely that they form a close pair.
\end{abstract}

Key words. galaxies: individual: NGC 185 - galaxies: stellar content - galaxies: structure

\section{Introduction}

This paper represents the latest installment of our carbon star survey of Local Group galaxies. The project started in order to collect homogeneous samples of $\mathrm{C}$ stars in galaxies of various morphological types and metallicities. The primary goal is to assess the universality of the photometric properties of the $\mathrm{C}$ star population in each galaxy. Although our effort has produced new data in the last three years, the observational Local Group data are still incomplete in many aspects: i) for some of the larger galaxies only a small fraction in area has been surveyed so far; ii) surveys have been conducted using different techniques, and some of the older surveys are incomplete in bolometric magnitude; iii) only for some galaxies there are information about the late M-star population, or these are sometimes unpublished even when the data are available; iv) not all galaxies in the Local Group have been surveyed; v) especially for some of the older works insufficient data are available to determine bolometric magnitudes (see Groenewegen 2002 for a recent review).

Send offprint requests to: $\mathrm{P}$. Battinelli, e-mail: battinel@oarhp1.rm.astro.it

* Based on observations carried out at the Canada-France-Hawaii Telescope, operated by the National Research Council of Canada, the Centre National de la Recherche Scientifique de France, and the University of Hawaii.
C stars, selected in a specific colour interval, do have a nearly constant $\left\langle M_{I}\right\rangle=-4.59$ in galaxies with at least one hundred such stars (Demers et al. 2003). However, Battinelli $\&$ Demers (2004), see also Nowotny et al. (2003), noted that the $\left\langle M_{I}\right\rangle$ of the C star population of NGC 147 is $3 \sigma$ fainter than this mean value. The fact that NGC 147 is the only spheroidal galaxy with an abundant intermediate-age population, in the sample of galaxies so far surveyed (Battinelli \& Demers 2004) urges us to investigate another galaxy of similar morphology, namely NGC 185 .

It soon, however, became evident that once identified, $\mathrm{C}$ stars can be quite useful to investigate the structure and population distribution in the outer parts of galaxies, as clearly shown by Letarte et al. (2002). In this respect, C stars offer two advantages over red giants: 1 - the Galactic contribution to the C star counts is essentially nil, indeed only one per $200 \mathrm{deg}^{2}$ late-giant C stars is observed by Totten \& Irwin (1998). Such stars would be brighter than their extragalactic counterparts, and none of them are dwarf carbon stars (Totten et al. 2000). This fact allows us to identify $\mathrm{C}$ stars in low density environment; 2- C stars are brighter, in the $I$ band, that the numerous old red giants seen in galaxies. They can then be detected with relatively short exposures, for example, less than $30 \mathrm{~min}$ of open-shutter time was required to observe NGC 185 . We note however that $\mathrm{C}$ star surveys in dwarf spheroidals is generally not very rewarding since the number $N_{\mathrm{C}}$ of $\mathrm{C}$ stars expected in 
these galaxy on the basis of the $M_{V, \text { gal }}-N_{\mathrm{C}}$ relation (Battinelli $\&$ Demers 2000) may be very low.

C star surveys employing a wide field mosaic have been successful in identifying proven galactic members located in the low density periphery. For example, Battinelli et al. (2003) have seen $\mathrm{C}$ stars up to the edge of the observed HI disk of M 31. These outer $\mathrm{C}$ stars can then be valuable kinematic probes to investigate the rotation or the mass distribution of galaxies.

The spheroidal galaxy NGC 185 is undoubtedly a satellite of M 31. Located at $\alpha(2000)=00^{\mathrm{h}} 40^{\mathrm{m}} 22^{\mathrm{s}}$ and $\delta=41^{\circ} 41^{\prime} 11^{\prime \prime}$ it is $7^{\circ}$ from M 31 . NGC 185 is at a relatively low Galactic latitude $\left(b=-14.5^{\circ}\right)$. Therefore its colour excess is far from negligible. Schlegel et al. (1998) obtain $E(B-V)=0.181$, from the COBE and IRAS maps at $100 \mu \mathrm{m}$. This corresponds to $E(R-I)=0.15$ and $A_{I}=0.28$ using the reddening ratios of Rieke \& Lebofsky (1985). Direct measurement of the colour excess of NGC 185 by Lee et al. (1993) yields a similar value: $E(B-V)=0.19 \pm 0.03$. We adopt this reddening.

One finds in the literature three independent distance determinations for NGC 185. From 151 RR Lyrae variables Saha \& Hoessel $(1990)$ obtained $(m-M)_{0}=23.79 \pm 0.25$ while Lee et al. (1993) and Martínez-Delgado \& Aparicio (1998) obtained respectively $23.96 \pm 0.21$ and $23.95 \pm 0.10$ from the tip of the red giant branch (TRGB). A re-evaluation of the TRGB method by Salaris \& Cassisi (1998) shifts the modulus of NGC 185 to 24.12, the distance adopted by van den Bergh (2000). This later determination would be our adopted distance for NGC 185. The mean metallicity of NGC 185 has been estimated from the colour of the red giant branch by Lee et al. (1993) $([\mathrm{Fe} / \mathrm{H}]=-1.23 \pm 0.16)$ and by Martínez-Delgado $\&$ Aparicio (1998) $([\mathrm{Fe} / \mathrm{H}]=-1.43 \pm 0.15)$. A spread of in the metallicity has been detected by these two studies. We shall assume that the $\mathrm{C}$ star population has a metallicity of $[\mathrm{Fe} / \mathrm{H}]=-1.33$, the average of both determinations.

An intermediate-age population has been known, for sometime, to exist in NGC 185 ever since Lee et al. (1993) noted the presence of numerous bright red stars. Their existence has recently been confirmed by the AGB survey of Nowotny et al. (2003). Using essentially the same narrow band technique that we employ, they identified 154 carbon stars in a $6.5^{\prime} \times 6.5^{\prime}$ area centered on NGC 185.

In the next section we describe our observations and the reduction procedure; in Sect. 3 the colour-magnitude and colour-colour diagrams are presented; in Sect. 4 we discuss the photometric properties of the $\mathrm{C}$ stars (Sect. 4.1), the C/M ratio (Sect. 4.2), the surface density profile of NGC 185 and its tidal radius (Sect. 4.3), the NGC 185-NGC 147 system (Sect. 4.4) and finally the mass determination of these two galaxies (Sect. 4.5).

\section{Observations}

The results presented here are based on observations obtained, in Service Queue observing mode in August 2002, with the CFH12K camera installed at the prime focus of the $3.66 \mathrm{~m}$ Canada-France-Hawaii Telescope. The camera consists in a $12000 \times 8000$ pixel mosaic covering a field of $42^{\prime} \times 28^{\prime}$, each
Table 1. Summary of the NGC 185 observations.

\begin{tabular}{llcc}
\hline \hline Filter & Exp. times & Average seeing (") & Mean airmass \\
\hline$R$ & $250 \mathrm{~s}$ & 0.96 & 1.138 \\
$I$ & $240 \mathrm{~s}$ & 0.79 & 1.139 \\
$\mathrm{CN}$ & $560 \mathrm{~s}$ & 0.64 & 1.337 \\
$\mathrm{TiO}$ & $560 \mathrm{~s}$ & 0.69 & 1.337 \\
\hline
\end{tabular}

pixel corresponding to 0.206 arcsec. Images were obtained through Mould $I$ and $R$ filters and narrowband $\mathrm{CN}$ and TiO filters, centered at $808.6 \mathrm{~nm}$ and $768.9 \mathrm{~nm}$, respectively. A summary of the acquired data is presented in Table 1 . The total exposure time to acquire the $\mathrm{C}$ star population was $27 \mathrm{~min}$, under sub-arcsec seeing.

The data distributed by the CFHT have been detrended. This means that the images have already been corrected with the master darks, biases, and flats. Fringes have been removed on $I$ exposures under $60 \mathrm{~s}$ and large scale structures such as the "Skyring" effect have been removed when relevant. This preanalysis produces $12 \mathrm{CCD}$ images, of a given mosaic, with the same zero point and magnitude scale.

The photometric reductions were done by fitting model point-spread functions (PSFs) using DAOPHOT-II/ALLSTAR series of programs (Stetson 1987, 1994). Instrumental magnitudes are calibrated using equations provided by the CFHT QSO team (see http://www.cfht.hawaii.edu/ Instruments/Elixir/stds.2003.05.html for details). The equations for $R$ and $I$ magnitudes are:

$$
\begin{aligned}
& R=26.190+m_{r}-0.09\left(X_{r}-1\right)+0.0094(R-I), \\
& I=26.185+m_{i}-0.04\left(X_{i}-1\right)-0.0511(R-I),
\end{aligned}
$$

where $X_{r}$ and $X_{i}$ are the airmasses of the $R$ and $I$ exposures. In term of instrumental magnitudes $r$ and $i$, obtained with DAOPHOT, and for the given exposure times, $m_{r}$ and $m_{i}$ correspond to:

$$
\begin{aligned}
& m_{r}=(r-25.0)+2.5 \log (250), \\
& m_{i}=(i-25.0)+2.5 \log (240) .
\end{aligned}
$$

By substracting the above $R$ and $I$ equations and using the appropriate airmasses we obtain:

$$
\begin{aligned}
& (R-I)=0.046+1.064(r-i), \\
& I=i+7.129-0.0511(R-I) .
\end{aligned}
$$

The zero point of the $(\mathrm{CN}-\mathrm{TiO})$ index is set according to the procedure outlined by Brewer et al. (1995). We set the mean of $(\mathrm{CN}-\mathrm{TiO})=0.0$ for all blue stars since hot stars are expected to have a featureless spectra in the $\mathrm{CN}$ and $\mathrm{TiO}$ regions. We thus define a blue star, as in Letarte et al. (2002), i.e. as a star in the colour range $0.0<(R-I)_{0}<0.45$.

\section{Results}

Figure 1 shows the colour-magnitude diagram of 50000 stars with photometric error $\sigma_{(R-I)}<0.10$. The colour location of 


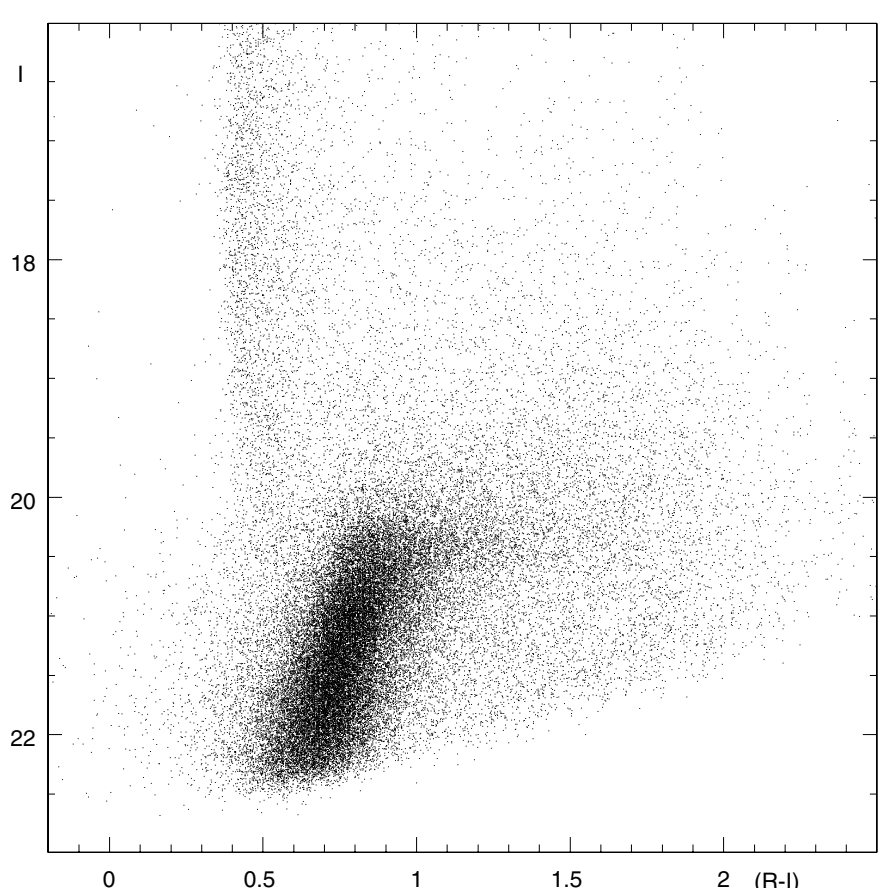

Fig. 1. Colour-magnitude diagram of the whole CFH12K field. Stars with colour errors $<0.10$ are plotted. We acquire the first 2.5 mag of the giant branch, quite sufficient to identify the $\mathrm{C}$ star population.

the vertical ridge, seen to reach $\approx 0.5$ at faint magnitudes, confirms our adopted reddening. This ridge corresponds to the field $\mathrm{G}$ dwarfs seen along the line of sight. The I-luminosity of the RGB tip, as determined by a Sobel convolution of the luminosity function, is $I_{\mathrm{TRGB}}=20.30 \pm 0.05$, in perfect agreement with Martínez-Delgado \& Aparicio (1998). We therefore assume that the uncertainty of the zero point of our photometry is \pm 0.05 mag.

Figure 2 presents the colour-colour diagram which allows the discrimination of $\mathrm{C}$ stars from $\mathrm{M}$ stars. Only stars with a combined error $\epsilon=\sqrt{\sigma_{(R-I)}^{2}+\sigma_{(\mathrm{CN}-\mathrm{TiO})}^{2}}$ smaller than $0.125 \mathrm{mag}$ are retained. Some 29000 stars are plotted. The two boxes define the regions of O-rich and C-rich stars as explained by Letarte et al. (2002). 145 stars fall into the $\mathrm{C}$ box, there are obviously more $\mathrm{C}$ stars, with bluer colours, but they will not be included in our sample. This number of $\mathrm{C}$ stars is low compared to galaxies of similar luminosity.

We list, in Table 2, the J2000.0 coordinates of the $\mathrm{C}$ stars identified along with their magnitude and colours. The $145 \mathrm{C}$ stars are identified, in the mosaic of the field in Fig. 3, by large dots. All carbon stars, excepted one are within 12 arcmin from the center of NGC 145. The one on the northern edge of the field has quite normal magnitude and colours, we see no reason to exclude it on the basis of the quality of its photometry.

\section{Discussion}

The presence of a number of bright bluish stars in the center of NGC 185, first noted by Baade a few years after his Local Group identification of NGC 185 (Baade 1944) demonstrates

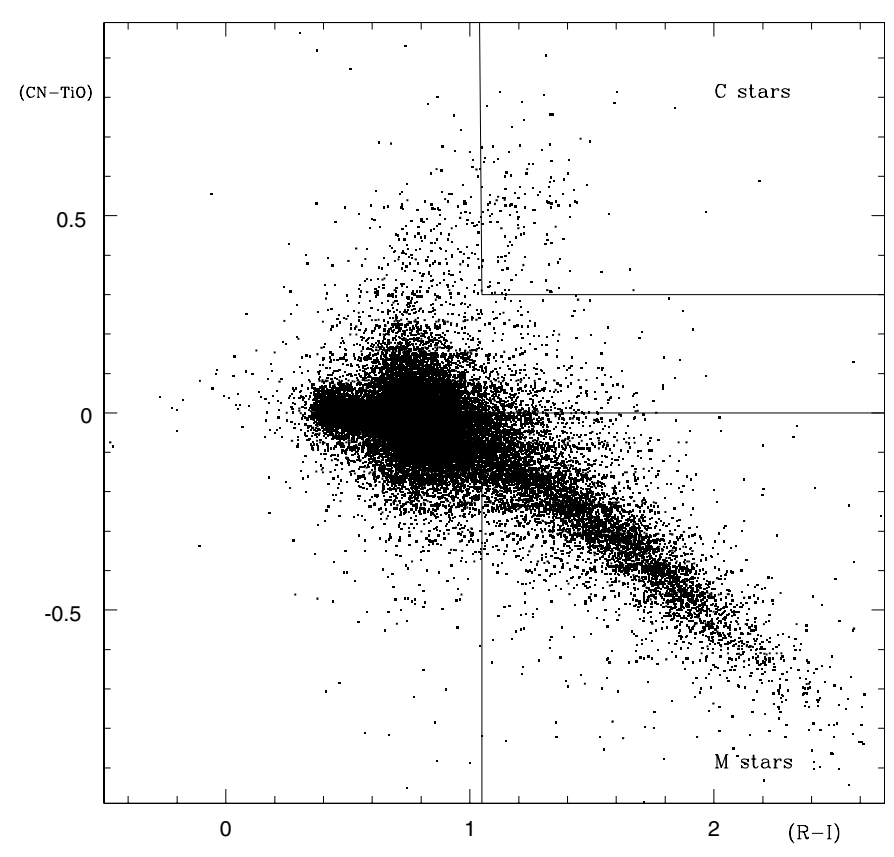

Fig. 2. Colour-colour diagram showing the boxes of $\mathrm{C}$ stars and $\mathrm{M}$ stars.

that NGC 185 is not made up of a pure old stellar population. The discovery of over one hundred RR Lyrae variables by Saha \& Hoessel (1990) suggested however that the bulk of NGC 185 is composed of old stars. This was confirmed by the deep CMD of Martínez-Delgado et al. (1999). Furthermore they interpret the CMD published by Martínez-Delgado \& Aparicio (1998) as proof of the presence of numerous intermediate age stars seen above the RGB. The direct identification of AGB stars was recently obtained by Nowotny et al. (2003). Our survey does indeed confirm that star formation in NGC 185 has occurred over an extended period of time.

\subsection{The photometric properties of $C$ stars}

The I magnitude distribution of the $145 \mathrm{C}$ stars is displayed in Fig. 4. As seen in other galaxies, the LF is Gaussian. Here, $\sigma=0.45$ a value $\sim 50 \%$ larger than seen in other galaxies. This scatter in magnitude could certainly be explained by the compactness of NGC 185 . The $\langle I\rangle=19.99 \pm 0.05$ corresponds to $\left\langle M_{I}\right\rangle=-4.41 \pm 0.05$ for the adopted distance and extinction and $\langle R-I\rangle=1.26 \pm 0.07$ thus $\left\langle(R-I)_{0}\right\rangle=1.11 \pm 0.07$. The mean absolute magnitude of the C stars of NGC 185 is essentially identical to the $\left\langle M_{I}\right\rangle$ of C stars in NGC 147 (Battinelli \& Demers 2004) both are fainter than the mean $-4.59 \pm 0.07$ determined for seven galaxies with numerous C stars (Demers et al. 2003). Da Costa (1994) explains the low luminosity of the two spectroscopically confirmed C stars in Phoenix $\left(M_{I}=-4.12,-3.95\right)$ by comparing their luminosities to the few $\mathrm{C}$ stars in Sculptor and Draco. He then concludes that the intermediate-age population of Phoenix must be quite old, $\sim 10$ Gyr. The two Phoenix $\mathrm{C}$ stars have $(R-I) \sim 0.80$ being warmer than our accepted blue limit, it is then quite reasonable for them to be fainter in $I$ mag. However, according to the models of Mouhcine \& Lançon (2003), only C stars younger than 
Table 2. C stars in NGC $185^{a}$.

\begin{tabular}{ccccccccc}
\hline \hline id & RA & Dec & $I$ & $\sigma_{I}$ & $R-I$ & $\sigma_{R-I}$ & $\mathrm{CN}-\mathrm{TiO}$ & $\sigma_{\mathrm{CN}-\mathrm{TiO}}$ \\
\hline 1 & $00: 38: 07.13$ & $48: 15: 45.70$ & 19.936 & 0.011 & 1.385 & 0.022 & 0.593 & 0.033 \\
2 & $00: 38: 20.06$ & $48: 20: 39.40$ & 20.248 & 0.016 & 1.226 & 0.028 & 0.520 & 0.050 \\
3 & $00: 38: 20.25$ & $48: 19: 27.90$ & 20.374 & 0.017 & 1.162 & 0.028 & 0.405 & 0.052 \\
4 & $00: 38: 23.43$ & $48: 18: 42.10$ & 20.841 & 0.023 & 1.134 & 0.038 & 0.363 & 0.084 \\
5 & $00: 38: 27.68$ & $48: 18: 59.90$ & 19.404 & 0.009 & 1.351 & 0.017 & 0.472 & 0.026 \\
6 & $00: 38: 28.61$ & $48: 18: 24.80$ & 20.209 & 0.015 & 1.083 & 0.023 & 0.533 & 0.044 \\
7 & $00: 38: 31.14$ & $48: 17: 45.30$ & 20.355 & 0.016 & 1.201 & 0.032 & 0.544 & 0.064 \\
8 & $00: 38: 34.97$ & $48: 18: 33.70$ & 20.302 & 0.022 & 1.296 & 0.039 & 0.667 & 0.043 \\
9 & $00: 38: 35.92$ & $48: 21: 32.30$ & 20.086 & 0.013 & 1.188 & 0.026 & 0.524 & 0.060 \\
10 & $00: 38: 38.52$ & $48: 16: 23.90$ & 20.058 & 0.013 & 1.454 & 0.023 & 0.695 & 0.039 \\
11 & $00: 38: 39.61$ & $48: 20: 03.10$ & 20.179 & 0.018 & 1.163 & 0.031 & 0.503 & 0.033 \\
12 & $00: 38: 39.67$ & $48: 18: 24.70$ & 20.407 & 0.023 & 1.409 & 0.043 & 0.498 & 0.056 \\
13 & $00: 38: 40.53$ & $48: 16: 24.40$ & 20.488 & 0.019 & 1.380 & 0.034 & 0.554 & 0.038 \\
14 & $00: 38: 41.29$ & $48: 17: 22.60$ & 20.172 & 0.018 & 1.091 & 0.028 & 0.504 & 0.052 \\
15 & $00: 38: 41.38$ & $48: 24: 12.70$ & 19.366 & 0.009 & 1.082 & 0.013 & 0.500 & 0.024 \\
\hline
\end{tabular}

${ }^{a}$ A portion of Table 2 is shown here for guidance regarding its form and content. Units of right ascensions are hours, minutes and seconds, and units of declination are degrees, arcminutes and arcseconds. The full table is only available in electronic form at the CDS via anonymous ftp to cdsarc.u-strasbg.fr (130.79.128.5) or via http://cdsweb.u-strasbg.fr/cgi-bin/qcat? J/A+A/417/479

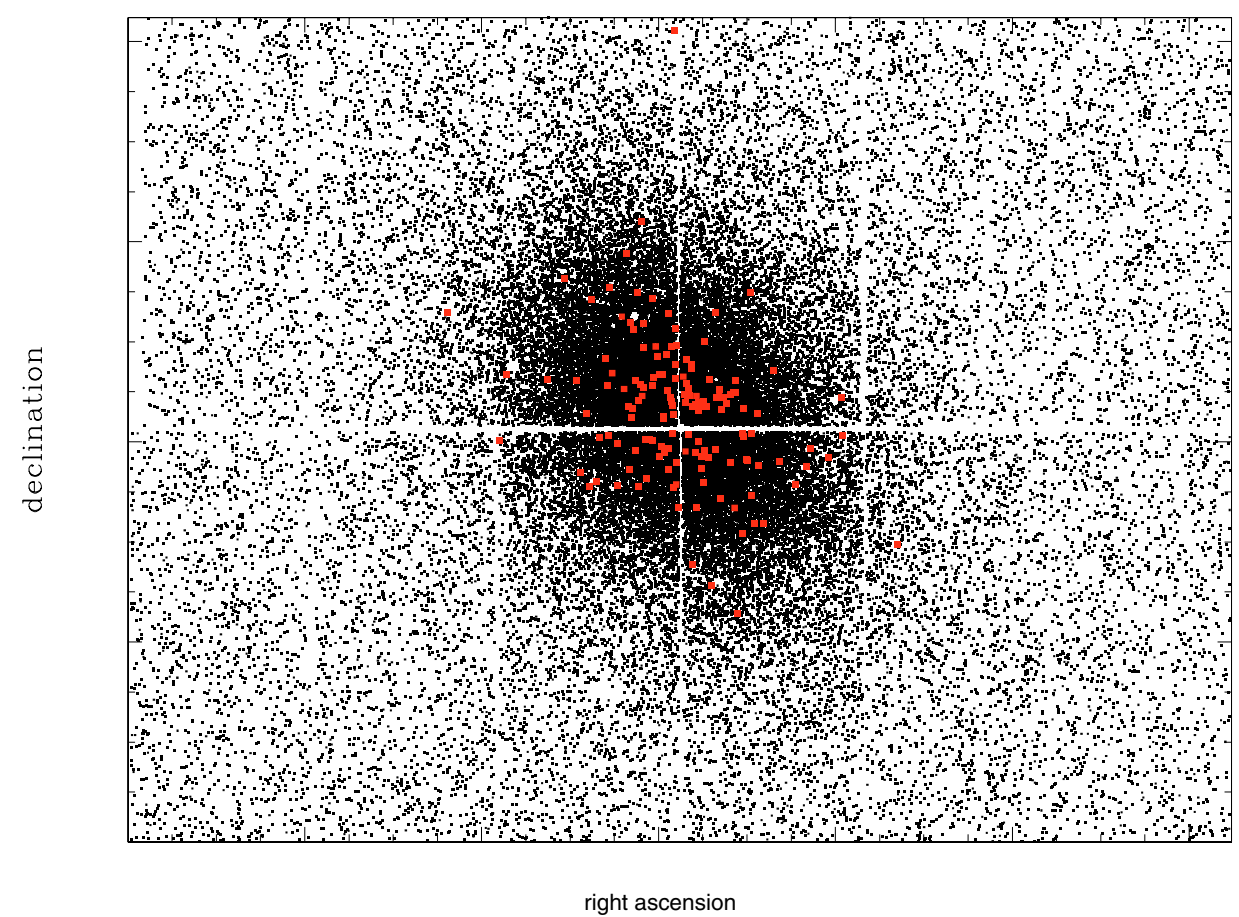

Fig. 3. The CFH12K field of NGC 185. Carbon stars identified in this study are represented by big dots. North is on top, East is on the left. The average gap, between the CCDs, in the $X$ direction is $7.8^{\prime \prime}$ while it is $6.8^{\prime \prime}$ in the $Y$ direction.

$1 \mathrm{Gyr}$ are expected to have a brighter luminosity than all the older C stars. NGC 185 does have a patch of blue stars in its center but the volume where stars are now forming is insignificant compared to the whole galaxy.

The lack of $\mathrm{C}$ stars younger than $1 \mathrm{Gyr}$ does not appear to us as the explanation for the lower luminosity of the $\mathrm{C}$ stars in
NGC 185 and NGC 147. In dwarf irregular galaxies, with ongoing star formation, one would certainly expect to see $\mathrm{C}$ stars of all ages. The C stars LF's in these galaxies are not wider than the LF's observed in the spheroidal galaxies. In the colour range considered, the spread of magnitude is the essentially the same. Furthermore, the apparent magnitudes of the $900 \mathrm{C}$ stars 


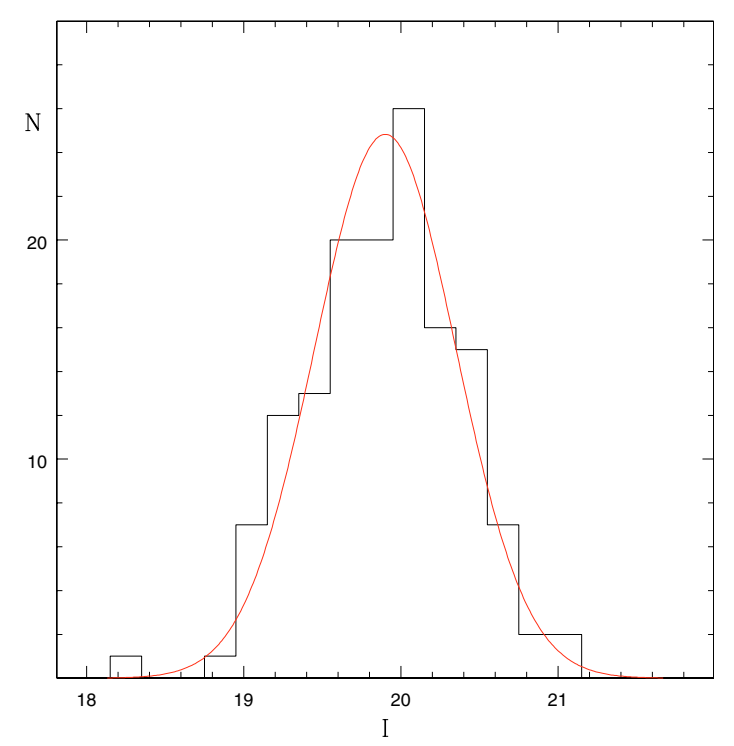

Fig. 4. Luminosity function of the C stars in NGC 185. An eye fitted Gaussian is traced over the histogram.

in NGC 6822 do not show a spatial trend which could be expected if numerous younger stars are located at the center where current star formation takes place.

One needs observations of other spheroidal galaxies with numerous $\mathrm{C}$ stars to investigate their LF. The $13 \mathrm{C}$ stars in the dwarf spheroidal galaxy Leo I have $\left\langle M_{I}\right\rangle=-4.79$ (Demers \& Battinelli 2002) a luminosity that goes contrary to what is seen in NGC 147 and NGC 185. It would be of interest to determine the $\left\langle M_{I}\right\rangle$ of the $\mathrm{C}$ star population of Fornax a galaxy with more $\mathrm{C}$ stars than Leo I. The C star population of the brightest dwarf spheroidals around M 31 may also reveal some clues as to the effect of the morphology on the $\left\langle M_{I}\right\rangle$ of C stars.

Recently, Nowotny et al. (2003) surveyed the AGB population of NGC 185 where they identified 154 carbon stars. Their definition of a $\mathrm{C}$ star is less restrictive than ours and consequently includes bluer $\mathrm{C}$ stars omitted by us. A cross identification (better than $1^{\prime \prime}$ ) of our C stars with theirs resulted in 32 matches. As noted before (Battinelli \& Demers 2004), their apparent $i$ magnitudes are $\sim 0.2$ mag fainter than our I magnitudes.

\subsection{The $\mathrm{C} / \mathrm{M}$ ratio}

The $\mathrm{C} / \mathrm{M}$ ratio of a stellar population is known to be function of the metallicity of that population (Battinelli \& Demers 2004; Groenewegen 1999). The number of C stars is relatively easy to obtain in a galaxy, but on the other hand the number of AGB M stars is much more difficult to properly assess because one has to know the contribution of the foreground $\mathrm{M}$ stars to the total counts.

To be consistent with our previous publications of this series and to follow Brewer et al. (1995), we select as AGB M stars a subset of the M stars (those in the M box of the colour-colour diagram) by applying a lower and upper magnitude cutoffs. $\mathrm{M}_{\mathrm{AGB}}$ must have their $M_{\text {bol }}<-3.5$ and the
$I>18.2$. The determination of the bolometric magnitude follows the procedure detailed by Battinelli et al. (2003).

The surface density of $\mathrm{M}_{\mathrm{AGB}}$ stars, belonging to NGC 185 , can be followed up to $12^{\prime}$ from the center. The density then reaches a plateau of $\rho=1.97 \pm 0.09$ stars $\mathrm{min}^{-2}$, number that we adopt for the foreground. Within the $12^{\prime}$ ellipse there are $144 \mathrm{C}$ stars and $1536 \mathrm{M}_{\mathrm{AGB}}$ stars. The estimated number of foreground stars in this area is 668 , a number to be substracted to the $\mathrm{M}_{\mathrm{AGB}}$ stars count. Thus, yielding a $\mathrm{C} / \mathrm{M}=0.17 \pm 0.02$. In the radial range from $0^{\prime}$ to $10^{\prime}$ there is little evidence for a radial gradient of the $\mathrm{C} / \mathrm{M}$ ratio.

\subsection{The stellar density profile of NGC 185}

Our C star survey of the Local Group galaxies is taking full advantage of the unusual wide field offered by the CFH12K camera. This opportunity allows not only to completely survey the $\mathrm{C}$ star population of nearby galaxies but also permits to investigate the extent of their stellar halo to unprecedented distances.

Hodge (1963) was the first to publish a survey of the structure of NGC 185. Eventhough he used Palomar Schmidt plates Hodge could not follow the profile further than a major axis distance of $300^{\prime \prime}$. He determined that the ellipticity $\epsilon=(1-b / a)$ of the galaxy increases slightly to reach 0.26 at a major axis distance of $6^{\prime}$. The position angle of the major axis was found to be $41^{\circ}$. Hodge (1963) determined also that the tidal limit of NGC 185 is $16 \pm 2^{\prime}$. The luminosity profile in the inner regions of NGC 185 has also been determined by Kent (1987), Lee et al. (1993) and Kim \& Lee (1998) they essentially confirm the results of Hodge.

To study the stellar surface distribution of NGC 185 we employ a file containing 49944 stars with good photometry and reaching $I \approx 22.2$. They are the stars plotted in Figs. 1 and 3 . We adopt the PA and $\epsilon$ as given by Hodge. We count stars in elliptical annulii centered on the galaxy. For major axis distances larger than $15^{\prime}$ the annulii spill outside of the CFH12K field. We then calculate the areas of sectors of elliptical annulii to properly determine the densities. With this approach, we can reach up to a major axis distance of $24^{\prime}$ when the ellipse touches the east and west borders of the field. The outer density profile is displayed in Fig. 5. The last four counts are averaged and are taken to represent the foreground surface density: 13.07 stars $\min ^{-2}$.

Using this estimated foreground density we plot, on Fig. 6, the logarithm of the density. The profile of NGC 185 follows a power law with a scale length of $\alpha=2.53 \pm 0.074^{\prime}$ corresponding to $500 \mathrm{pc}$ at $667 \mathrm{kpc}$, the adopted distance. This value is larger than the $1.5^{\prime}$ quoted by Caldwell et al. (1992) it is however smaller than the scale length of NGC 147, recently obtained by Battinelli \& Demers (2004). The density profile of NGC 185 does not fit a de Vaucouleurs' law as $r^{1 / 4}$. The $\mathrm{C}$ stars, plotted as open circles on Fig. 6, have a smaller scale length than the old halo population of NGC 185. A least square fit through the points yields a scale length of $1.56 \pm 0.06^{\prime}$ demonstrating that the intermediate-age population of NGC 185 is more concentrated than the old population. 


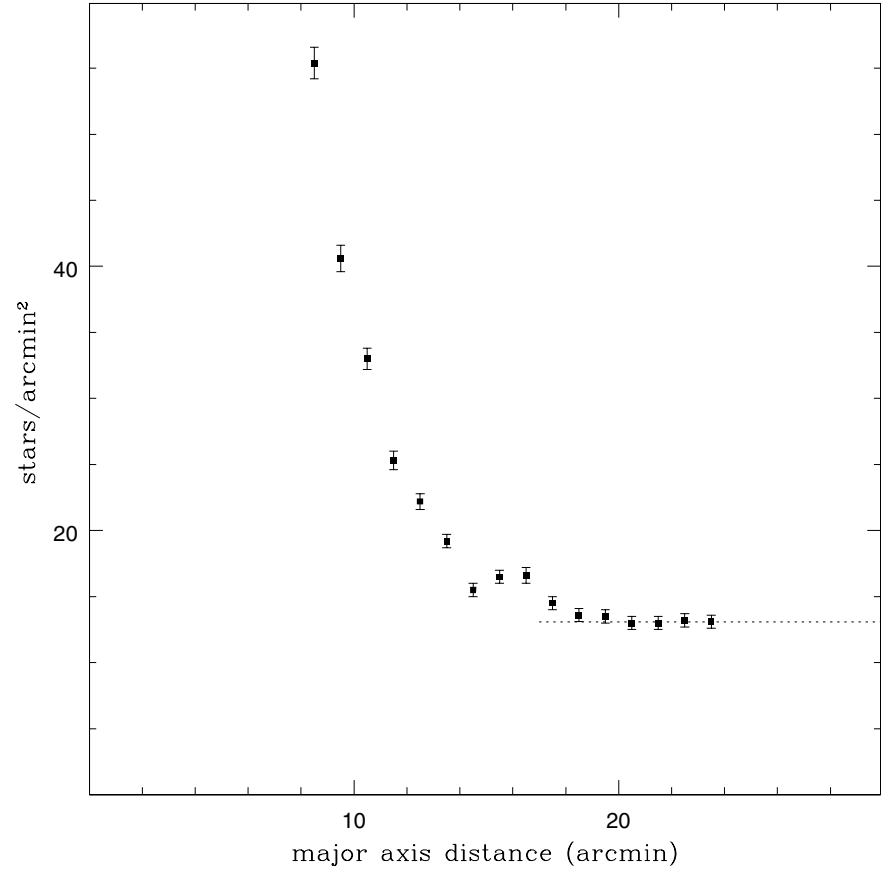

Fig. 5. Surface density profile of the outer parts of NGC 185 . The foreground is reached at $\sim 19^{\prime}$.

Martínez-Delgado et al. (1999) state that the relative amount of stars of different ages is similar in each regions of NGC 185. Figure 6 shows that, within the area surveyed by them $\left(r<6^{\prime}\right)$, the density profile of $\mathrm{C}$ stars appears essentially similar to the one of old giants. When the $\mathrm{C}$ star profile is plotted over longer distances, the different slope becomes obvious. Harbeck et al. (2001) have recently studied the population gradients in some Local Group dwarf spheroidals finding metallicity driven gradients in Sextans, Sculptor, Tucana and Andromeda IV, while for Carina the age is the major factor determining the observed population gradients. For NGC 185 the different scale lengths we found for the old and intermediate-age stars, along with the lack of evidence supporting a clear $\mathrm{C} / \mathrm{M}$ gradient suggest that the population gradients in this galaxy are, similarly to Carina, mainly age driven.

A formal King tidal radius can also be obtained for NGC 185 by fitting the last points of the profile in a $\sqrt{\rho}$ versus $1 / r$ plot. This relation, displayed in Fig. 7 , yields a tidal radius $r_{\mathrm{t}}=22.5 \pm 2.2^{\prime}$. NGC 185 fits nicely the $r_{\mathrm{t}}$ vs. $\alpha$ relation determined by Bingelli \& Cameron (1991) for Virgo cluster dwarf elliptical galaxies. When compared to this sample of Virgo dEs, NGC 185 has, however, a small scale length for its absolute magnitude.

\subsection{Do NGC 147 and NGC 185 really form a bound system?}

van den Bergh (1998) has demonstrated than NGC 185 forms a pair with NGC 147, barely located one degree from each other. This interpretation hinges on the adopted distances of both galaxies to the Sun and is based on the fact that the radial velocities of both galaxies differ by barely $9 \mathrm{~km} \mathrm{~s}^{-1}$. The currently

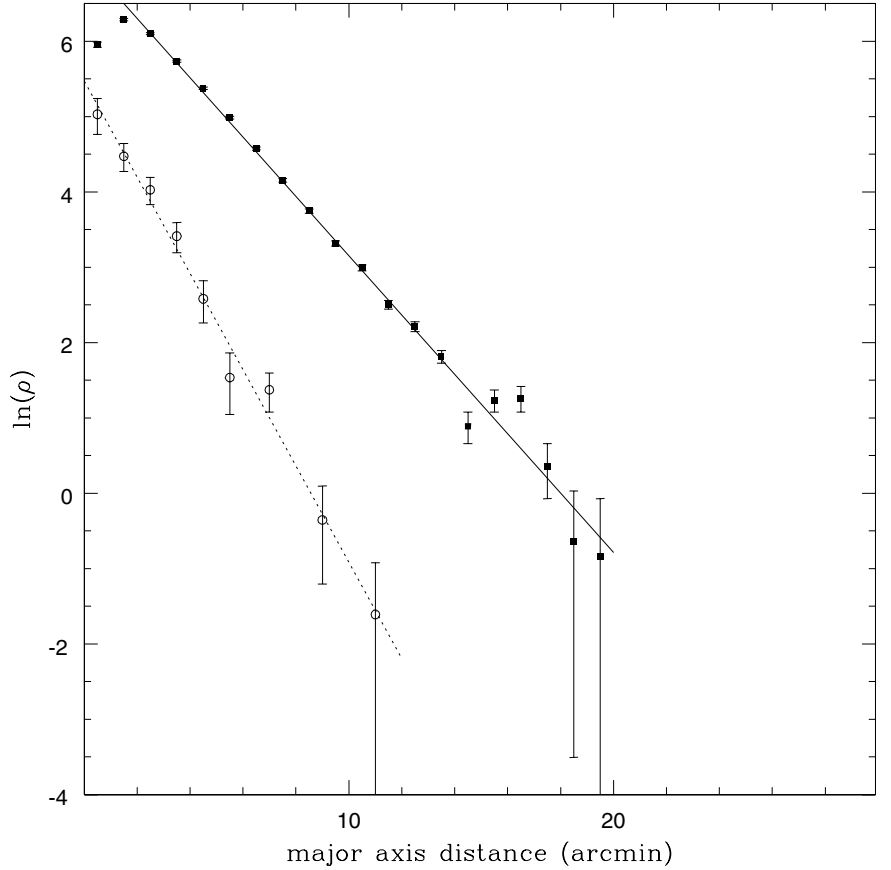

Fig. 6. The density profile of the halo red giant stars follows a power law. The density profile of $\mathrm{C}$ stars (where counts have been multiplied by 20 to facilitate comparison), represented by open circles. The two lines correspond to the least square fits of the two profiles.

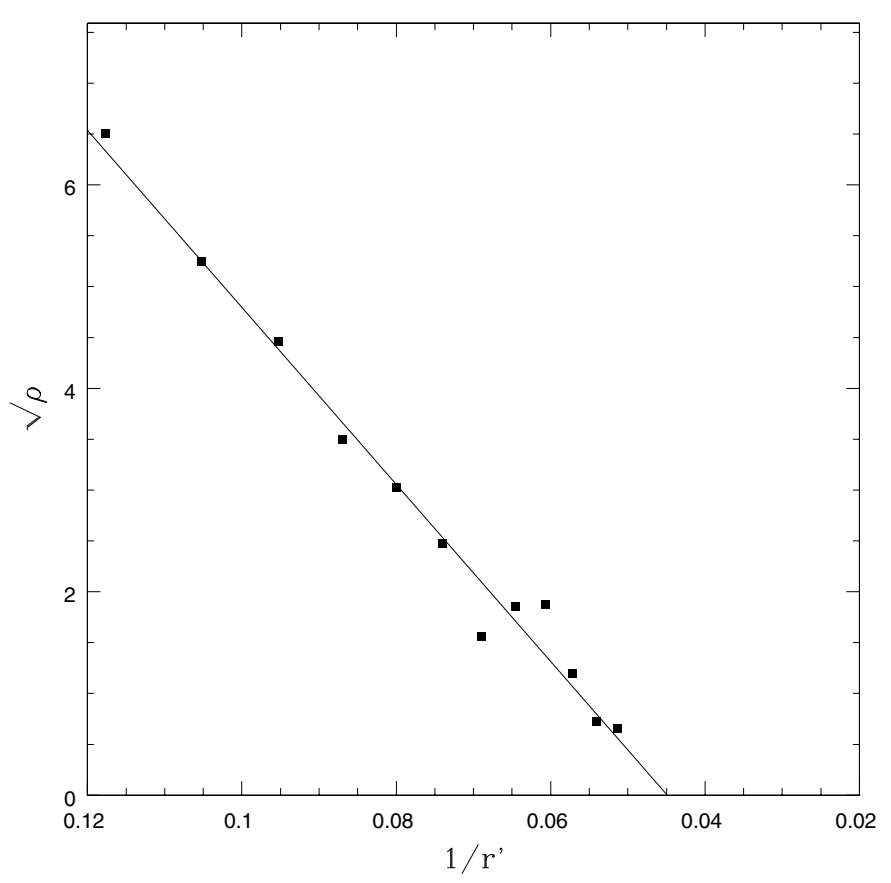

Fig. 7. Surface density profile of the outer parts of NGC 185 yields a tidal radius of $22.5 \pm 2.2^{\prime}$.

"accepted" distances for these galaxies are, however, not identical. Mateo (1998) puts NGC $147100 \mathrm{kpc}$ further away than NGC 185. More recently, Tonry et al. (2001) determined the distance of NGC 147 and NGC 185, along with 300 galaxies, using the surface brightness fluctuation technique. They found 


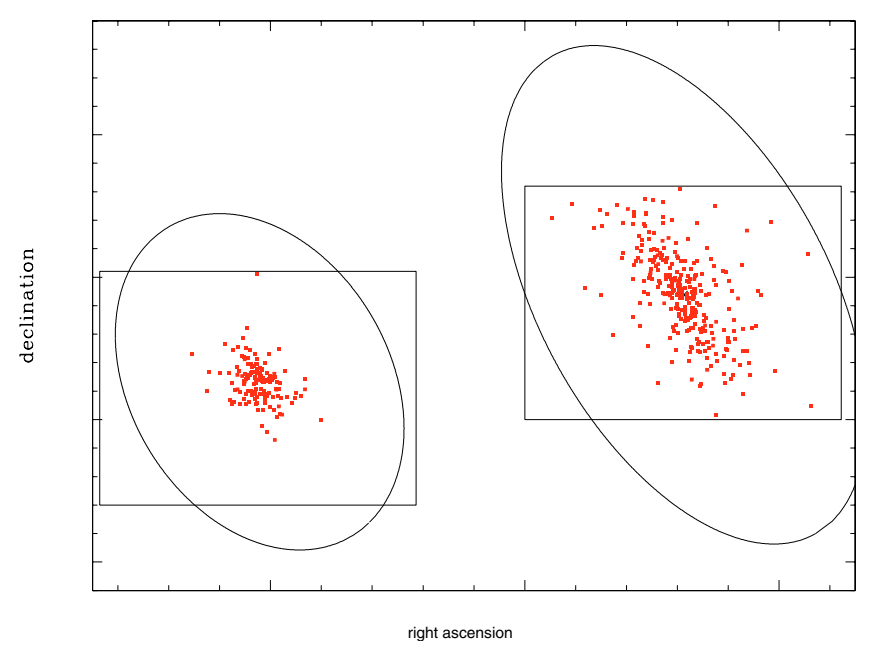

Fig. 8. A $100^{\prime} \times 76^{\prime}$ map of the C star populations of NGC 185 , on the left, and NGC 147, on the right. The ellipses represent their tidal radius, the two rectangles are the $\mathrm{CFH} 12 \mathrm{~K}$ fields. The centers of the two galaxies are just one degree apart. East is on the left North at the top.

respectively $24.44 \pm 0.16$ and $24.02 \pm 0.16$ thus confirming that NGC 147 and NGC 185 are not near each other. Nowotny et al. (2003) AGBs survey also confirms the distance difference of $\sim 0.3 \mathrm{mag}$. Our own C star results $\langle I\rangle=19.99 \pm 0.05$, for NGC 185 and $\langle I\rangle=20.31 \pm 0.05$ (Battinelli \& Demers 2004) for NGC 147 prove that these two galaxies are not near each other. Their reddenings are essentially identical.

Carbon stars, being some Gyr old, may have been witnesses of past interactions. They are then excellent candidates to provide clues of the gravitational "stirring up" of galaxies. One may thing, for example, of the inter Magellanic Cloud region where a number of C stars have been seen (Demers et al. 1993). Figure 8 presents, to scale, the two galaxies with their $\mathrm{C}$ star population. The ellipses represent their tidal radius. The two rectangles correspond to the $\mathrm{CFH} 12 \mathrm{~K}$ surveyed fields. There is currently a gap between the two halos. If the two galaxies had been closer or in interaction, it is then reasonable to expect a tidal bridge between them. A tenuous stellar bridge will be hard to detect, their Galactic latitudes not being particularly high. On the other hand, C stars can be detected, irrespectively of their number since they are seen behind a Galactic foreground of zero. Observation of a $9^{\prime} \times 9^{\prime}$ field did not reveal any $\mathrm{C}$ stars (Battinelli \& Demers 2004). We therefore conclude that all evidences point to a case of optical binary galaxies.

\subsection{Mass estimates for NGC 185 and NGC 147}

Assuming that the two galaxies are gravitationally bound, van den Bergh (1998) calculated a lower limit for their combined mass from the difference in their radial velocity, he obtains $2.7 \times 10^{8} M_{\odot}$. Bender et al. (1991) determined the central velocity dispersion of both galaxies to be $23 \mathrm{~km} \mathrm{~s}^{-1}$. With the luminosity profiles they obtained the $\mathrm{M} / \mathrm{L}$ ratios.

Using the newly determined surface density profiles of NGC 185 and NGC 147 (Battinelli \& Demers 2004) we can, following Rood et al. (1972), (their Eq. (2)), obtain an estimates of their central density, from their core radius $r_{\mathrm{c}}$ and their line of sight velocity dispersion. $r_{\mathrm{c}}$, the radius where the surface density is half the central value is easily measured to be 2.82' (619 pc) for NGC 147 and 1.75' (346 pc) for NGC 185. Assuming that both galaxies are prolate spheroids, the central mass is obtained by multiplying the central density by the volume limited to $r_{\mathrm{c}}$. We find $1.3 \times 10^{8} M_{\odot}$ for NGC 185 and $0.6 \times 10^{8} M_{\odot}$ for NGC 147 , yielding rather low M/L ratios close to one.

\section{Conclusion}

NGC 185 and NGC 147 both satellites of M 31 have the same luminosity (Mateo 1998) but this is essentially their only similarity. Detailed analysis of their stellar populations has shown that they differ markedly in many aspects. Contrary to NGC 147, NGC 185 contains gas, dust and a population of blue stars in its center (Martínez-Delgado et al. 1999). NGC 147 appears almost completely dust and gas free (Young \& Lo 1997; Sage et al. 1998; Young 1999). Furthermore, the chemical composition of NGC 147 and NGC 185 are not identical, their mean $[\mathrm{Fe} / \mathrm{H}]$ being respectively -0.9 (Han et al. 1997) and -1.3 , our adopted value.

Our C star surveys have revealed that both galaxies possess a large tenuous stellar halo whose stellar populations are well mixed in NGC 147 but are not well mixed in NGC 185. Indeed, we show that the radial scale length of the intermediate-age population of NGC 185 is significantly smaller, indicating that the younger stars are more concentrated than the older ones. According to Bender et al. (1991) both galaxies have the same velocity dispersion, within a radius of $100^{\prime \prime}$. NGC 147 seems to rotate while NGC 185 does not. Obviously, now that bright members of both galaxies have been positively identified as far as $15^{\prime}$ from their center, the kinematics of individual stars is the next logical step to endeavour to understand the population distribution.

Acknowledgements. This research is funded in parts (S.D.) by the Natural Science and Engineering Council of Canada.

\section{References}

Baade, W. 1944, ApJ, 100, 147

Battinelli, P., \& Demers, S. 2000, AJ, 120, 1807

Battinelli, P., \& Demers, S. 2004, A\&A, in press

Battinelli, P., Demers, S., \& Letarte, B. 2003, AJ, 125, 1298

Bender, R., Paquet, A., \& Nieto, J.-L. 1991, A\&A, 246, 349

Bingelli, B., \& Cameron, L. 1991, A\&A, 252, 27

Brewer, J. P., Richer, H. B., \& Crabtree, D. R. 1995, AJ, 109, 2480

Caldwell, N., Armandroff, T. E., Seitzer, P., \& Da Costa, G. S. 1992, AJ, 103, 840

Demers, S., \& Battinelli, P. 2002, AJ, 123, 238

Demers, S., Dallaire, M., \& Battinelli, P. 2002, AJ, 123, 3428

Demers, S., Battinelli, P., \& Letarte, B. 2003, A\&A, submitted

Demers, S., Irwin, M. J., \& Kunkel, W. E. 1993, MNRAS, 260, 103

Da Costa, G. S. 1994, in Dwarf Galaxies, Proc. ESO/OHP Workshop, ed. G. M. Meylan, \& P. Prugnel (Garching ESO), ESO Conf. Workshop Proc., 49, 221 
Groenewegen, M. A. T. 1999, in Asymptotic Giant Branch Stars, ed. T. Le Bertre, A. Lebre, \& C. Waelkens, IAU Symp., 191, 535

Groenewegen, M. A. T. 1999, Ringberg Castle Workshop, [arXiv: astro-ph/0208449]

Han, M., Hoessel, J. G., Gallagher, J. S., III, Holtzman, J., \& Stetson, P. B. 1997, AJ, 113, 1001

Harbeck, D., Grebel, E. K., Holtzman, J., et al. 2001, AJ, 122, 3092

Hodge, P. W. 1963, AJ, 68, 691

Kent, P. C. II 1987, AJ, 94, 306

Kim, S. C., \& Lee, M. G. 1998, JKAS, 31, 51

Lee, M. G., Freedman, W. L., \& Madore, B. F. 1993, AJ, 106, 964

Letarte, B., Demers, S., Battinelli, P., \& Kunkel, W. E. 2002, AJ, 123 , 832

Martínez-Delgado, D., \& Aparicio, A. 1998, AJ, 115, 1462

Martínez-Delgado, D., Aparicio, A., \& Gallart, C. 1999, AJ, 118, 2229

Mateo, M. 1998, ARA\&A, 36, 435

Mouhcine, M., \& Lançon, A. 2003, MNRAS, 338, 572

Nowotny, W., Kerschbaum, F., Olofsson, H., \& Schwarz, H. E. 2003, A\&A, 403, 93
Rieke, G. M., \& Lebofsky, M. J. 1995, ApJ, 288, 618

Rood, H. J., Page, T. L., Kintner, E. C., \& King, I. R. 1972, ApJ, 175, 627

Sage, L. J., Welch, G. A., \& Mitchell, G. F. 1998, ApJ, 507, 726

Saha, A., \& Hoessel, J. G. 1990, AJ, 99, 97

Salaris, M., \& Cassisi, S. 1998, MNRAS, 289, 166

Schlegel, D., Finkbeiner, D., \& Davis, M. 1998, ApJ, 500, 525

Stetson, P. B. 1987, PASP, 99, 191

Stetson, P. B. 1994, PASP, 106, 250

Tonry, J. L., Dressler, A., Blakeslee, J. P., et al. 2001, ApJ, 546, 681

Totten, E. J., \& Irwin, M. J. 1998, MNRAS, 294, 1

Totten, E. J., Irwin, M. J., \& Whitelock, P. A. 2000, MNRAS, 314, 630

van den Bergh, S. 1998, AJ, 116, 1688

van den Bergh, S. 2000, The Galaxies of the Local Group (Cambridge: Cambridge University Press), 35

Young, L. M. 1999, ASP Conf. Ser., 163, 109

Young, L. M., \& Lo, K. Y. 1997, ApJ, 476, 127 